\title{
Chromatographic and Computational Studies on the Chiral Recognition of Sulfated $\beta$-Cyclodextrin on Enantiomeric Separation of Milnacipran
}

Pranav Pathak ${ }^{\text {a }}$, Vikalp Jain ${ }^{\text {a }}$, Elvis A. F. Martis ${ }^{\text {b }}$, Evans C. Coutinho ${ }^{\text {b }}$, Krishnapriya Mohanraj*a

${ }^{a}$ Department of Pharmaceutical Analysis, Bombay College of Pharmacy, Kalina, Santacruz (East), Mumbai- 400098, India

${ }^{\mathrm{b}}$ Molecular Simulations Group, Department of Pharmaceutical Chemistry, Bombay College of Pharmacy, Kalina, Santacruz (East), Mumbai- 400098, India

*Address for correspondence: Department of Pharmaceutical Analysis, Bombay College of Pharmacy, Kalina, Santacruz (East), Mumbai- 400098, India. Email: krishnapriyamohanraj@gmail.com

\section{Highlights:}

1. This is the first report of the enantiomeric separation of milnacipran hydrochloride with RPHPLC using sulfated $\beta$-cyclodextrin, as a chiral mobile phase additive.

2. Molecular docking studies for chiral recognition mechanism.

3. A cost-effective method as separation time was limited to 5 minutes with the help of the coreshell column. 


\begin{abstract}
A new, cost-effective and fast chromatographic method using sulfated $\beta$-cyclodextrin $(\mathrm{S} \beta C D)$ as a chiral mobile phase additive (CMPA) was developed and validated for the enantiomeric separation of milnacipran. Milnacipran is an anti-depressant drug. Levo-milnacipran is the active enantiomer with less adverse effects than dextro-milnacipran. Hence, it is imperative to separate the enantiomers of milnacipran. Various parameters affecting enantiomeric resolution, for instance, the effect of type and concentration of cyclodextrins, the effect of $\mathrm{pH}$ of the mobile phase, effect of type and concentration of the organic solvent in the mobile phase and effect of type of achiral column, were investigated. We demonstrated successful resolution of enantiomers of milnacipran on reverse-phase HPLC with core-shell Kinetex $\mathrm{C}_{8}$ column $(150 \times 4.6 \mathrm{~mm}, 5 \mu)$, using a mobile phase consisting of 18:82 v/v acetonitrile: $10 \mathrm{mM}$ sodium dihydrogen orthophosphate dihydrate buffer $\mathrm{pH} 3.0$ (adjusted with orthophosphoric acid) containing 10mM S $\beta C D$ with a flow rate $1.0 \mathrm{ml} /$ minute. The column temperature was ambient and UV detection was carried out at 227 $\mathrm{nm}$ with an injection volume of $20 \mu 1$. This method for enantiomeric separation of milnacipran was validated in accordance with ICH guidelines and successfully applied to the marketed formulation of Levo-milnacipran. Furthermore, molecular docking was used to identify the chiral recognition mechanism. The results of molecular docking corroborated with our experimental findings.
\end{abstract}

\title{
Keywords:
}

Enantiomeric separation, Reversed phase-HPLC, Chiral mobile phase additive, Milnacipran hydrochloride, Sulfated $\beta$-cyclodextrin, Molecular docking. 


\section{Introduction}

Milnacipran is an antidepressant drug that belongs to the class of serotonin and norepinephrine reuptake inhibitors (SNRIs). It shows better selectivity than serotonin for blocking the norepinephrine reuptake [1]. Milnacipran hydrochloride $( \pm)-[1 \mathrm{R}(\mathrm{S}), 2 \mathrm{~S}(\mathrm{R})]-2-($ aminomethyl)N,N-diethyl-1-phenylcyclopropane-1-carboxamide hydrochloride has two chiral centres (Figure 1). Levo-milnacipran is the active enantiomer of milnacipran and was approved for the treatment of the major depressive disorder (MDD) by the US Food and Drug Administration (FDA) in 2013. Its efficacy is significantly higher than racemic milnacipran with fewer risks of cardiovascular disturbances and organ/tissue toxicity [2]. Hence, it is imperative to separate enantiomers of milnacipran.

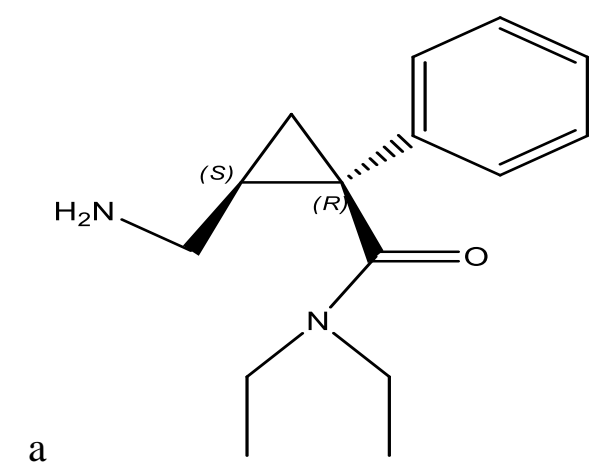

. $\mathrm{HCl}$

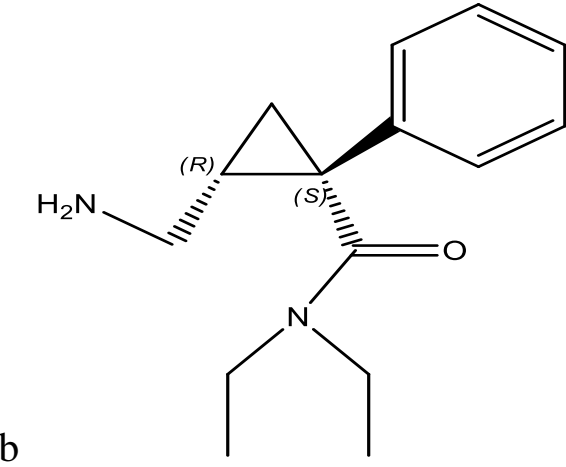

. $\mathrm{HCl}$

\section{Figure 1: Structures of (a) (1R, 2S) - milnacipran hydrochloride (Dextro) and (b) (1S, 2R) - milnacipran hydrochloride (Levo)}

Ever since the Thalidomide disaster in 1960, the US-FDA guidelines made it mandatory to evaluate properties of each enantiomer and racemates separately, and chiral switches for marketing off-patent drugs as a single enantiomer are the driving forces for increased marketing of single enantiomers of chiral drugs. Therefore, it is very important to separate enantiomers and to quantify the drug and chiral impurity. This requires a powerful means of enantiomeric separation and detection. [3, 4] The commonly used technique for chiral separation is High-Performance Liquid Chromatography (HPLC). Direct techniques utilize chiral stationary phases (CSPs) or chiral mobile phase additives (CMPA), and indirect techniques involve chiral derivatization [5]. In the CMPA technique, an enantiomerically pure compound (chiral mobile-phase additive) is added to the HPLC mobile phase. Reversed-phase HPLC is used to separate the enantiomers. This method offers several advantages like low cost of separation attributed to relatively inexpensive achiral stationary phases, absence of any derivatization step and improved flexibility compared to CSPs. 
Cyclodextrins have been widely used as chiral selectors [5,6]. Owing to the internal hydrophobic cavity and external hydrophilic rim, cyclodextrins form inclusion complexes with hydrophobic molecules and enhance the dissolution rate by significantly reducing the non-polar surface area. $\beta$ cyclodextrin $(\beta C D)$, Hydroxypropyl $\beta$-cyclodextrin $(\mathrm{H} \beta C D)$, Sulfated $\beta$-cyclodextrin $(\mathrm{S} \beta C D)$ were investigated as chiral mobile phase additives for the resolution of milnacipran enantiomers.

The survey of literature revealed that there are chromatographic methods for enantiomeric separation of chiral drugs using $\beta$-cyclodextrin as chiral mobile phase additives [7-14]. But there are no reported methods for enantiomeric separation of milnacipran using chiral mobile phase additives. Enantiomers of milnacipran have been separated on chiral columns, hydroxypropyl $\beta$ cyclodextrin-based chiral column Astec CYCLOBOND ${ }^{\text {TM }}$ I 2000 RSP column in reversed-phase mode with resolution of 1.63[15], acetyl- $\beta$-cyclodextrin-based chiral column Astec CYCLOBOND ${ }^{\mathrm{TM}}$ I 2000 AC in reversed-phase mode with resolution of 1.74[16], Chiralcel OJ in normal-phase mode [17] and by a nonchiral HPLC - circular dichroism detector [18] has been reported.

In view of these facts, efforts were made to develop a cost-effective chiral HPLC method for enantiomeric separation of milnacipran using reverse phase column and $\beta$-cyclodextrin and its derivatives as chiral mobile phase additives. Since the method will be developed without using costly chiral stationary phases or costly chiral detector, the cost of the analysis will be significantly reduced. The aim of the present study is to separate and quantify the enantiomers of milnacipran using chiral mobile phase additives and to elucidate the chiral recognition mechanism.

\section{MATERIALS AND METHODS}

\subsection{Chemicals and reagents}

Racemic and Levo-milnacipran hydrochloride was obtained as a gift sample from Arch Pharma Labs, Mumbai, India. All the chemicals used, i.e. sodium dihydrogen orthophosphate dihydrate HPLC grade and orthophosphoric acid AR grade were purchased from S.D. Fine Chem Pvt. Ltd, Mumbai, India. Methanol and acetonitrile HPLC grade were purchased from Merck Chem Ltd., Mumbai, India. Chiral mobile phase additives such as $\beta$-cyclodextrin and hydroxypropyl $\beta$ cyclodextrin were obtained as gift samples from Signet Chemical Corporation, Mumbai, India and sulfated $\beta$ - cyclodextrin (degree of substitution 7-11) was purchased from Sigma Aldrich and synthesized in-house (Manuscript under process). Levo-milnacipran hydrochloride capsules 
(Fetzima, 120mg) manufactured by Forest Pharmaceuticals, INC. USA were used for assay studies.

\subsection{Instrumentation and chromatographic conditions}

The HPLC system consists of Jasco 2000 HPLC, intelligent binary HPLC pump, PU-2080 plus, equipped with a $20 \mu 1$ loop. The detector used was Intelligent UV/Vis detector UV-2075. Data acquisition and processing were performed using BORWIN software. The chromatographic columns used during method development were Kinetex $\mathrm{C}_{8}(150 \mathrm{~mm}$ x $4.6 \mathrm{~mm}$ I.D., $5 \mu \mathrm{m})$, Hyperclone $\mathrm{C}_{8}(150 \mathrm{~mm} \times 4.6 \mathrm{~mm}$ I.D., $5 \mu \mathrm{m})$, and HiQsilC 8 (250 mm x $4.6 \mathrm{~mm}$ I.D., $\left.5 \mu \mathrm{m}\right)$. The flow rate of the mobile phase was $1.0 \mathrm{ml} /$ minute and the ambient column temperature was maintained. The eluent was monitored at a wavelength of $227 \mathrm{~nm}$.

\subsection{Preparation of the mobile phase}

Acetonitrile and $10 \mathrm{mM}$ sodium dihydrogen orthophosphate dihydrate buffer $\mathrm{pH} 3.0$ (adjusted with orthophosphoric acid) containing $10 \mathrm{mM}$ S $\beta C D$ were used as mobile phase components. The mobile phase was filtered through a $0.45 \mu \mathrm{m}$ durapore nylon filter and sonicated for 5 minutes before use.

\subsection{Preparation of sample solution}

Stock solutions of the racemic milnacipran and Levo-milnacipran were prepared by dissolving 20 mg of each drug in $20 \mathrm{ml}$ acetonitrile separately. Working standard solutions were prepared by suitable dilutions of primary stock solution with the mobile phase.

\subsection{Preparation of solutions for precision}

For precision 15, 30, and 45 ppm concentration solutions of racemic milnacipran were used (7.5, 15, and 22.5 ppm for each enantiomer). 1000 ppm stock solution of racemic milnacipran was used for preparing various concentrations. Aliquots of racemic milnacipran standard stock solution $(1000 \mathrm{ppm})$ of 150,300 , and $450 \mu \mathrm{l}$ were transferred in three different $10 \mathrm{ml}$ volumetric flasks and diluted with the mobile phase to make 15,30, and 45 ppm respectively. All solutions were pipetted out by $100-1000 \mu$ micropipette.

\subsection{Preparation of solutions for accuracy}

For accuracy study target concentration for racemic milnacipran was chosen as $30 \mathrm{ppm}$ (15 ppm for each enantiomer). Accuracy test was performed at three concentration level i.e. 50\%, 100\%, and $150 \%$. For accuracy 7.5, 15, and 22.5 ppm concentration solutions of Levo-milnacipran were used. The volume of racemic milnacipran and Levo-milnacipran required for accuracy study at 
different concentration level are mentioned in Table 1. Aliquots of racemic milnacipran and Levomilnacipran standard stock solution $(1000 \mathrm{ppm})$ were transferred in three different $10 \mathrm{ml}$ volumetric flasks and diluted with the mobile phase.

Table 1: Volume of racemic milnacipran and Levo-milnacipran for accuracy studies

\begin{tabular}{|c|c|c|c|}
\hline $\begin{array}{l}\text { Concentration level } \\
\text { (For levomilnacipran) }\end{array}$ & $\begin{array}{l}\text { Volume of } 100 \mu \mathrm{g} / \mathrm{ml} \\
\text { racemic milnacipran } \\
(\mu \mathrm{l})\end{array}$ & $\begin{array}{l}\text { Volume of } 100 \mu \mathrm{g} / \mathrm{ml} \\
\text { levomilnacipran }(\mu \mathrm{l})\end{array}$ & $\begin{array}{l}\text { Final concentration } \\
\text { of levomilnacipran } \\
(\mu \mathrm{g} / \mathrm{ml})\end{array}$ \\
\hline $50 \%(7.5 \mathrm{ppm})$ & 1000 & 250 & 7.5 \\
\hline $100 \%(15 \mathrm{ppm})$ & 1000 & 1000 & 15 \\
\hline $150 \%(22.5 \mathrm{ppm})$ & 1000 & 1750 & 22.5 \\
\hline
\end{tabular}

\subsection{Assay of Levo-milnacipran hydrochloride in the formulation}

20 capsules of Levo-milnacipran hydrochloride were weighed and powder equivalent to $25 \mathrm{mg}$ Levo-milnacipran hydrochloride was accurately weighed and transferred to a $25 \mathrm{ml}$ standard volumetric flask. $20 \mathrm{ml}$ of acetonitrile was added to the flask and sonicated for $30 \mathrm{~min}$, and volume was made up using the same solvent. An aliquot of the solution was filtered through a $0.45 \mu \mathrm{m}$ nylon filter and $150 \mu \mathrm{l}$ transferred to a $10 \mathrm{ml}$ standard volumetric flask to yield a concentration of $15 \mu \mathrm{g} / \mathrm{ml}$.

\subsection{Molecular Docking to estimate complexation stability}

The coordinates of $\beta C D$ were imported from the protein data bank co-crystallized with glycosyltransferase (PDB id 3CGT). The protein around $\beta C D$ was deleted to obtain the coordinates of $\beta C D$. The $3 \mathrm{D}$ model of $\mathrm{S} \beta \mathrm{CD}$ was constructed by modifying the $\mathrm{CH}_{2} \mathrm{OH}$ groups at the $6^{\text {th }}$ position of the glucose moieties of $\beta \mathrm{CD}$ with $\mathrm{SO}_{3}{ }^{1-}$ group to maintain the degree of substitution as 7. The GLIDE module in Schrödinger Suite 2015 was used for docking the drug in the S $\beta C D$ cavity. The grid for docking was prepared by defining the centre of mass $(\mathrm{COM})$ of the host molecules (considering all atoms) and the dimensions of the inner grid box was fixed at $15 \AA$ x $15 \AA$ x $15 \AA$ and $20 \AA$ x $20 \AA$ x $20 \AA$ for the outer grid box to allow enough sampling space. Prior to docking, drugs were optimized to correct their geometry using the LIGPREP module in Schrödinger Suite 2015 using the OPLS 2005 force field. During docking, the van der Waals radii for the hosts and the guest molecule were scaled to $0.60 \AA$ to soften the potential over the nonpolar areas that lie within the grid extents and the partial atomic charges were set to 0.25 (default). The GLIDE Emodel and the standard-precision scoring function were employed to rank the docking solutions. 


\section{RESULTS AND DISCUSSION}

\subsection{Chiral HPLC Method development}

To achieve enantiomeric separation of milnacipran, factors affecting enantiomeric resolution were investigated, which include the effect of types and concentration of $\beta$-cyclodextrins, the effect of $\mathrm{pH}$, effect of organic solvent, and the effect of type of achiral column.

\subsubsection{Effect of types and concentration of $\beta$-cyclodextrins}

The method development was started with the addition of $\beta-\mathrm{CD}$ in the aqueous mobile phase. 10 and $15 \mathrm{mM}$ were the concentrations of $\beta-\mathrm{CD}$ used. The saturation solubility of $\beta-\mathrm{CD}$ in water is $15 \mathrm{mM}$, and hence, a higher concentration of $\beta-\mathrm{CD}$ could not be used. Since the limited solubility of $\beta-C D$ in water was the major limitation. $\mathrm{Hp}-\beta-\mathrm{CD}$ was used as CMPA, because it has good solubility in water therefore, it can be used at higher concentrations. 10,15 , and $25 \mathrm{mM}$ were the concentration of Hp- $\beta-C D$ used. Then $10 \mathrm{mM}$ S- $\beta-\mathrm{CD}$ was employed as CMPA. The results of trials taken with $\beta-\mathrm{CD}, \mathrm{Hp}-\beta-\mathrm{CD}$, and S- $\beta-\mathrm{CD}$ as CMPA at various concentrations and mobile phase compositions are mentioned in Table 2.

Table 2: The results of trials taken with $\beta-C D, H p-\beta-C D$, and $S-\beta-C D$ as CMPA

\begin{tabular}{|c|c|c|c|c|c|}
\hline S. N. & CMPA & $\begin{array}{c}\text { Mobile phase } \\
\text { ACN: Buffer }\end{array}$ & $\begin{array}{c}\text { Retention time } \\
\text { (Minutes) }\end{array}$ & Resolution & $\begin{array}{c}\text { Run time } \\
\text { (Minutes) }\end{array}$ \\
\hline 1 & $10 \mathrm{mM} \beta-C D$ & $20: 80$ & 19.8 & - & 25 \\
\hline 2 & $10 \mathrm{mM} \beta-C D$ & $10: 90$ & $85.08 \& 88.06$ & 1.2 & 100 \\
\hline 3 & $15 \mathrm{mM} \beta-C D$ & $10: 90$ & $46.76 \& 47.87$ & 0.89 & 52 \\
\hline 4 & $10 \mathrm{mM} \mathrm{Hp}-\beta-C D$ & $20: 80$ & 19.94 & - & 22 \\
\hline 5 & $10 \mathrm{mM} \mathrm{Hp}-\beta-C D$ & $10: 90$ & $62.06 \& 64.47$ & 1.14 & 70 \\
\hline 6 & $15 \mathrm{mM} \mathrm{Hp}-\beta-C D$ & $10: 90$ & $49.7 \& 52$ & 1.24 & 57 \\
\hline 7 & $25 \mathrm{mM} \mathrm{Hp}-\beta-C D$ & $10: 90$ & $31.8 \& 33.4$ & 1.06 & 38 \\
\hline 8 & $10 \mathrm{mM} \mathrm{S-} \beta-C D$ & $10: 90$ & $38.33 \& 48.62$ & 6.41 & 52 \\
\hline 9 & $10 \mathrm{mM} \mathrm{S-} \beta-C D$ & $20: 80$ & $9.16 \& 10.15$ & 2.98 & 12 \\
\hline 10 & $10 \mathrm{mM} \mathrm{S-} \beta-C D$ & $30: 70$ & 4.4 & 1.05 & 6 \\
\hline
\end{tabular}

Mobile phase: Acetonitrile: $10 \mathrm{mM}$ sodium dihydrogen orthophosphate dihydrate buffer CMPA pH 3.0 adjusted with

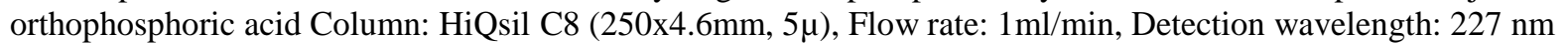

From results, one pattern was observed. Whenever the concentration of CMPA was increased, retention times decreased. As the concentration of CMPA increased, the polarity of diastereomeric complex increased and complex will not be retained on the non-polar $\mathrm{C} 8$ column, which affects resolution. When $\beta-C D$ was used, $10 \mathrm{mM} \beta-\mathrm{CD}$ gave a resolution of 1.2 in the run-time of 100 minutes. When HP- $\beta$-CD was used, $15 \mathrm{mM}$ HP- $\beta$-CD gave a resolution of 1.24 in the run-time 
of 57 minutes. Enantiomers of milnacipran were successfully resolved when S- $\beta$-CD was used as CMPA. Initially, trials were taken on a fully porous reversed-phase silica column, HiQsil C8 $(250 \times 4.6 \mathrm{~mm}, 5 \mu)$. The mobile phase composition was found to be important for enantioselectivity. The resolution between enantiomers and retention times increased as the aqueous phase containing CMPA was increased. A good resolution of 2.98 between milnacipran enantiomers was obtained with a mobile phase comprising acetonitrile: buffer (20:80). The run time was 12 minutes. In order to further optimize the method, a core-shell silica column was used.

\section{Core-shell silica column:}

Core-shell silica columns have solid core covered with a porous layer or shell of a silica adsorbent. The solid core plus the porous shell provides columns with speed and efficiency with low operating back pressure and it also reduces band broadening thus improving resolution. The porous shell provides less band broadening by reducing the dispersion of the solute molecules within a packed bed owing to a lower pore volume available for longitudinal diffusion (B term in the van Deemter equation-Eq.1). A shorter diffusion path length because of solid core, diminishes the contribution of the $\mathrm{C}$ term to band broadening due to the fast mass transfer. The contribution to A term (column packing regularity) is significantly reduced, because of a rougher surface of core-shell particles [19]. In equation $1, \mathrm{H}$ is height equivalent to the theoretical plate, $\mathrm{A}$ is eddy diffusion, $\mathrm{B}$ is longitudinal diffusion, $\mathrm{C}$ is mass transfer and $\mu$ is linear velocity

$$
\mathrm{H}=\mathrm{A}+\mathrm{B} / \mu+\mathrm{C} \mu \ldots \ldots \ldots(\mathrm{Eq} .1)
$$

Two achiral HPLC columns were investigated for enantiomeric resolution. One was a column with

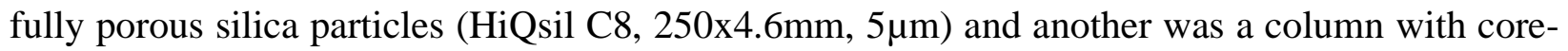

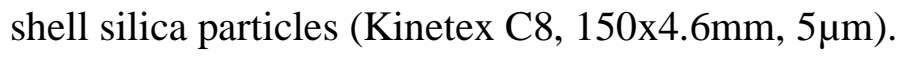

From the comparison between two achiral columns, huge disparity was observed in the retention times and resolution. The probable parameters were the change in the length of the column and core-shell particles. Hence, to identify the cause, further studies were conducted on another fully porous silica column i.e. Hyperclone $\mathrm{C}_{8} 150 \mathrm{mmx} 4.6 \mathrm{~mm}, 5 \mu \mathrm{m}$. The same chromatographic conditions were applied for enantiomeric separation of milnacipran hydrochloride on Hyperclone $\mathrm{C}_{8}$ Column. The results obtained are shown in Table 3 and Figure 2. 


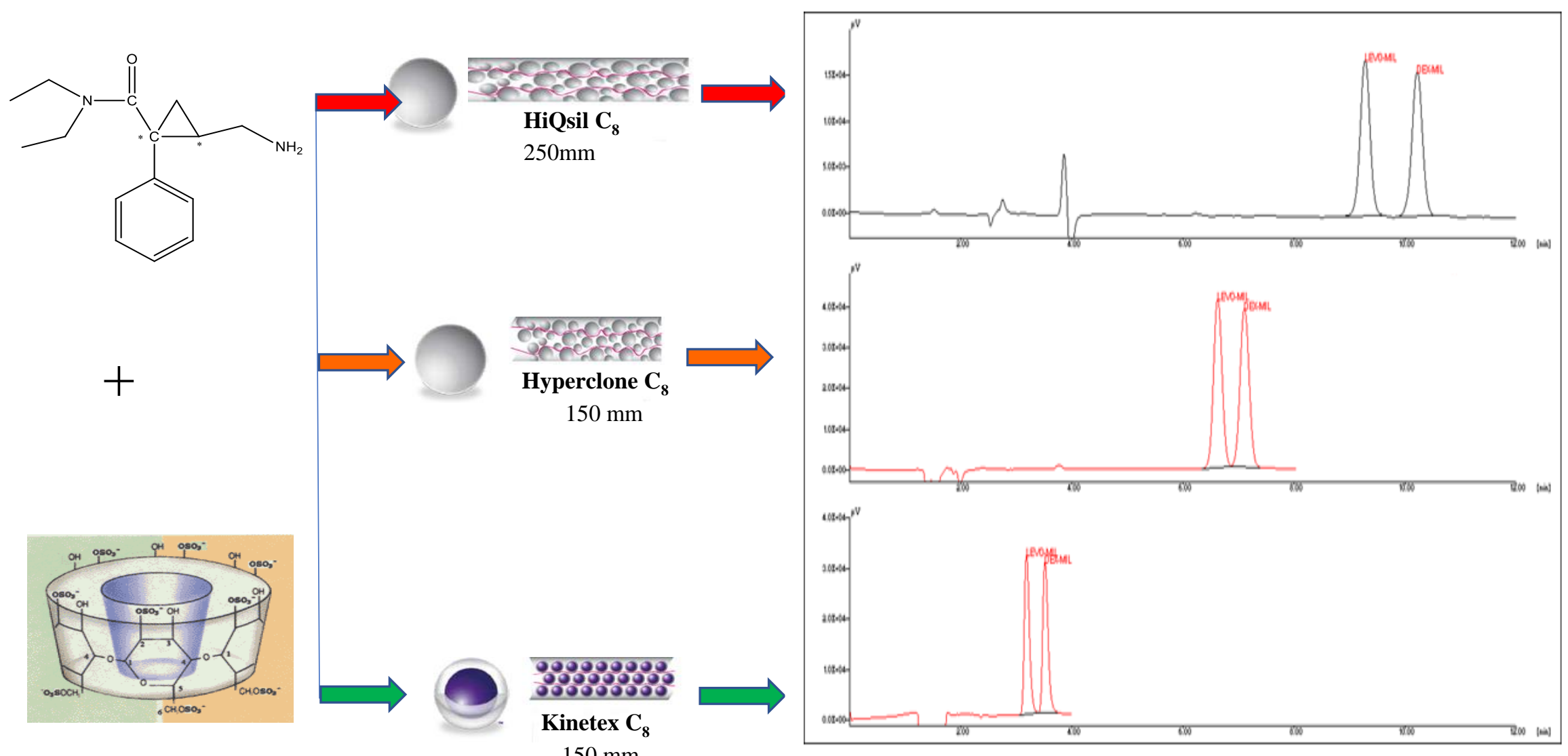

$150 \mathrm{~mm}$

Figure 2: Comparison of chromatographic performances of three achiral columns

Levo-Mil=Levo-milnacipran, Dex-Mil= Dextro-milnacipran

Chromatographic conditions: Mobile phase: Acetonitrile: $10 \mathrm{mM}$ sodium dihydrogen orthophosphate dihydrate buffer $10 \mathrm{mMS}-\beta-\mathrm{CD}$ pH 3.0 adjusted with orthophosphoric acid (20:80), Flow rate: 1ml/min, Detection wavelength: $227 \mathrm{~nm}$ 
Table 3: Comparison of chromatographic performances of three achiral columns

\begin{tabular}{|c|c|c|c|c|}
\hline S. N. & Achiral Column & $\begin{array}{c}\text { Retention time } \\
\text { (Minutes) }\end{array}$ & Resolution & $\begin{array}{c}\text { Run time } \\
\text { (Minutes) }\end{array}$ \\
\hline 1 & $\begin{array}{c}\text { HiQsil C } \text { Column }_{2} \\
(250 \mathrm{mmx} 4.6 \mathrm{~mm}, 5 \mu)\end{array}$ & $9.1 \& 10.1$ & 2.98 & 12 \\
\hline 2 & $\begin{array}{c}\text { Hyperclone } \mathrm{C}_{8} \text { Column } \\
(150 \mathrm{mmx} 4.6 \mathrm{~mm}, 5 \mu)\end{array}$ & $6.7 \& 7.1$ & 1.76 & 4 \\
\hline 3 & $\begin{array}{c}\text { Kinetex } \mathrm{C}_{8} \text { Column } \\
(150 \mathrm{mmx} 4.6 \mathrm{~mm}, 5 \mu)\end{array}$ & $3.2 \& 3.5$ & 2.48 & 4 \\
\hline
\end{tabular}

Mobile phase: Acetonitrile: $10 \mathrm{mM}$ sodium dihydrogen orthophosphate dihydrate buffer $10 \mathrm{mM} \mathrm{S-} \beta$-CD pH 3.0 adjusted with orthophosphoric acid (20:80), Flow rate: $1 \mathrm{ml} / \mathrm{min}$, Detection wavelength: $227 \mathrm{~nm}$

From these results, it was observed that the variations in retention times and resolution were not only due to change in length of the column but also due to the core-shell particles i.e. Kinetex $\mathrm{C}_{8}$ column. In the case of Hyperclone C8, the resolution between enantiomers was not good though retention times were higher than Kinetex C8. This can be attributed to peak width since it is inversely proportional to resolution (Eq.2). In Hyperclone C8, peaks are wide hence reducing resolution and in kinetex $\mathrm{C} 8$ peaks are sharp hence good resolution is obtained.

$$
\mathrm{Rs}=2(\mathrm{t} 2-\mathrm{t} 1) /(\mathrm{W} 1+\mathrm{W} 2) \ldots \ldots \ldots \ldots(\text { Eq. } 2)
$$

It is beneficial to use a core-shell column because all the system suitability parameters were satisfied with less analysis time. The consumption of the chiral selector (Sulfated- $\beta-C D$ ) was less when the core-shell column was used. Hence, the method with the core-shell column can be considered cost-effective. Therefore, it was decided to develop the method on the Kinetex C8 column.

\subsubsection{Optimization of concentration of sulfated $\beta$-cyclodextrin:}

The concentration of $\mathrm{S} \beta \mathrm{CD}$ had a prominent effect on retention time and ultimately on the resolution of milnacipran enantiomers. 8 and $10 \mathrm{mM}$ concentrations of S $\beta C D$ were investigated. At $8 \mathrm{mM}$ concentration, we observed an increased retention time with a decrease resolution. On the other hand, at $10 \mathrm{mM}$ concentration of $\mathrm{S} \beta C D$, the resolution was better with relatively less retention time seen. This can be attributed to enhanced host-guest complex formation between $\mathrm{S} \beta \mathrm{CD}$ and milnacipran enantiomers due to higher S $\beta C D$ concentration. The polar diastereomeric complex formed was not retained on the non-polar stationary phase. Therefore, $10 \mathrm{mM}$ of S $\beta C D$ was considered optimum. The Mobile phase was acetonitrile: 10mM sodium dihydrogen phosphate buffer 10mM S- $\beta$-CD pH 3.0 adjusted with orthophosphoric acid (20:80). 


\subsubsection{Optimization of $\mathrm{pH}$ of the mobile phase}

The $\mathrm{pH}$ of the mobile phase is one of the important parameters governing the resolution of enantiomers. It was observed that the best separation was obtained when the $\mathrm{pH}$ of the mobile phase was kept 3. Although, the retention times were nearly the same for $\mathrm{pH} \mathrm{3,4}$ and 6., the resolution was best $(\mathrm{Rs}=2.48)$ with $\mathrm{pH} 3$. This was because, at $\mathrm{pH} 4$ and $\mathrm{pH} 6$, wider peaks were observed and hence resolution was decreased. The result obtained with different $\mathrm{pH}$ of the mobile phase is shown in Figure 3.

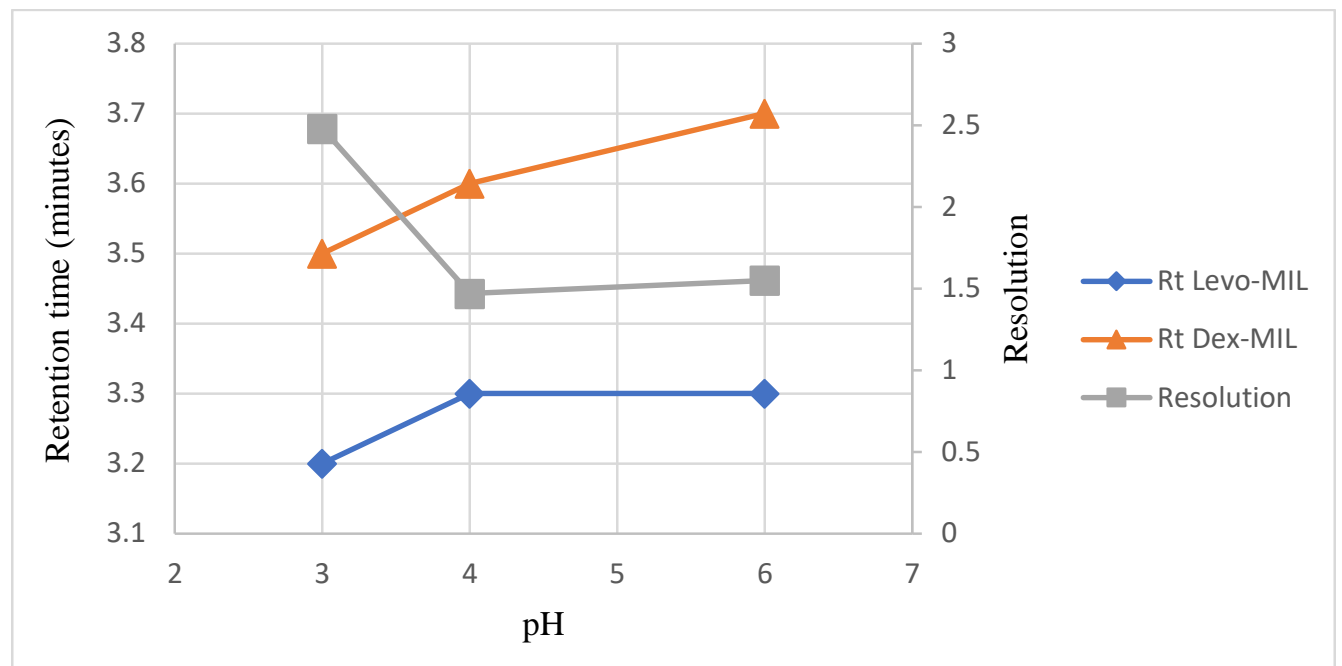

Figure 3: Effect of $\mathrm{pH}$ on retention times and resolution

Chromatographic conditions: Mobile phase: Acetonitrile: $10 \mathrm{mM}$ sodium dihydrogen orthophosphate dihydrate buffer $10 \mathrm{mM} \mathrm{S}-\beta-\mathrm{CD}$ pH $(3,4,6)$

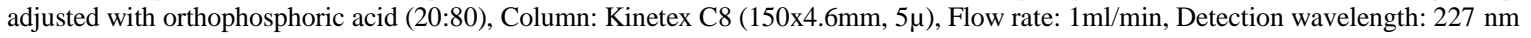

\subsubsection{Optimization of the organic solvent}

The effect of concentrations of methanol on retention times and resolution were studied. Instead of $20 \%$ acetonitrile, $20 \%$ methanol was used as an organic solvent, but retention times were quite high with high resolution. On using 30\% Methanol, retention times decreased with a satisfactory resolution. But due to high column back-pressure and increased retention times with methanol as compared to acetonitrile, acetonitrile was selected as an organic solvent. With $20 \%$ acetonitrile in mobile resolution between enantiomers was 2.48. In order to increase resolution, $18 \%$ acetonitrile was tried, which increased retention times and resolution (2.96).

\section{Final Developed HPLC Method:}

The final optimized mobile phase was acetonitrile: $10 \mathrm{mM}$ sodium dihydrogen orthophosphate dihydrate buffer, $\mathrm{pH} 3.0$ adjusted with orthophosphoric acid with 10mM Sulfated 
$\beta$ cyclodextrin as chiral mobile phase additive. The order of elution was determined by injecting a single enantiomer. The Levo-milnacipran eluted early and Dextro-milnacipran eluted later. The chromatogram of the optimized method is shown in Figure 4. The system suitability parameters for the final developed method are mentioned in Table 4.

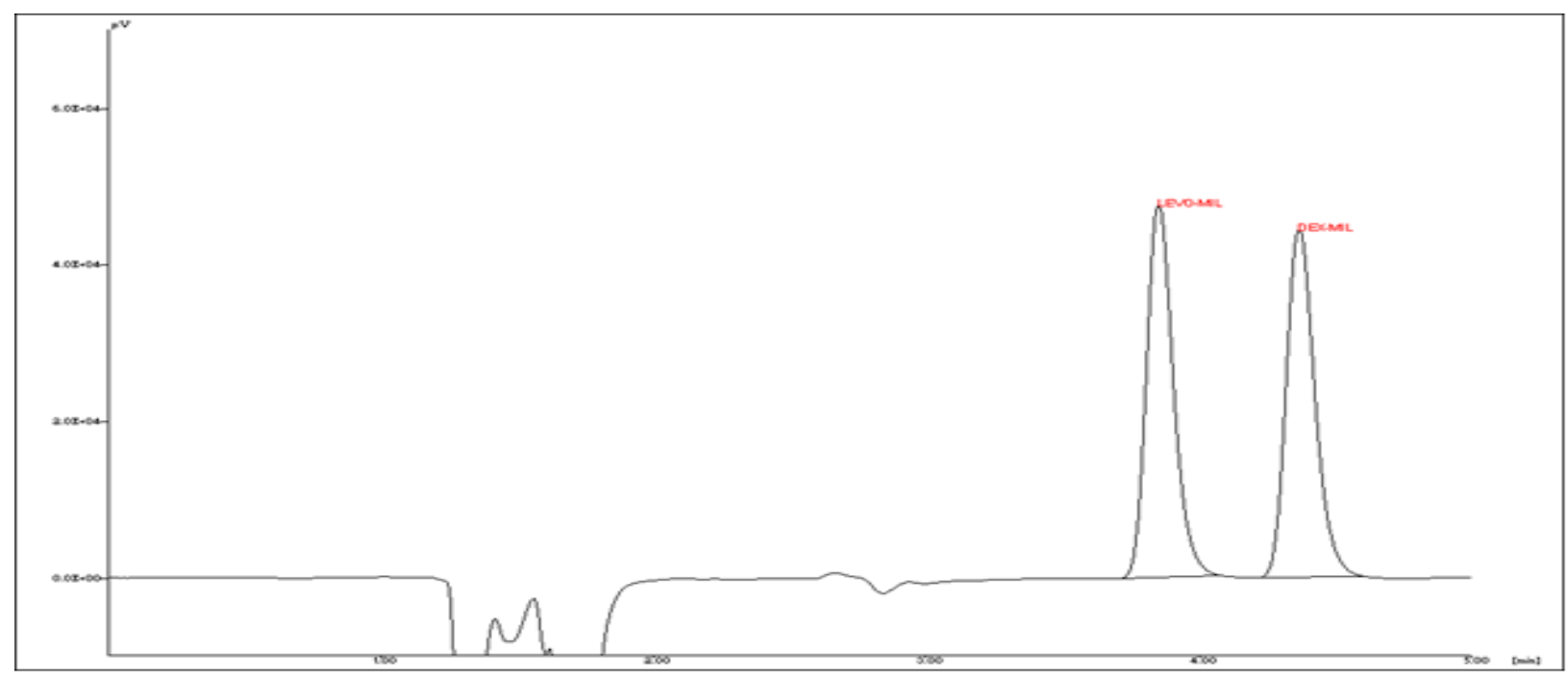

Levo-Mil = Levo-milnacipran , Dex-Mil = Dextro-milnacipran

Chromatographic conditions: 1) Mobile Phase: Acetonitrile: 10mM sodium dihydrogen orthophosphate dihydrate Buffer containing 10mM sulfated $\beta-\mathrm{CD}, \mathrm{pH} 3.0,(18: 82 \mathrm{v} / \mathrm{v})$; 2) Column: Kinetex $\mathrm{C}_{8}, 150 \mathrm{~mm}$ x $4.6 \mathrm{~mm}, 5 \mu$. 3) Column temperature: Ambient 4) Flow rate: $1 \mathrm{ml} /$ minute; 5) Detection wavelength: $227 \mathrm{~nm}$

Figure 4: Enantiomeric separation of racemic milnacipran hydrochloride

Table 4: System suitability parameters

\begin{tabular}{|l|c|c|c|c|}
\hline Enantiomers & $\begin{array}{l}\text { Retention times } \\
\text { (minutes) }\end{array}$ & Resolution & $\begin{array}{l}\text { Number of } \\
\text { theoretical plates }\end{array}$ & $\begin{array}{l}\text { Symmetry } \\
\text { Factor }\end{array}$ \\
\hline \hline Levo -Mil & 3.85 & - & 8365.27 & 1.25 \\
\hline Dex -Mil & 4.36 & 2.96 & 9330.82 & 1.26 \\
\hline
\end{tabular}

Levo-Mil=Levo-milnacipran , Dex-Mil= Dextro-milnacipran

\subsection{Method Validation:}

After optimization of the parameters affecting the enantiomeric resolution of milnacipran, the method was validated for linearity, the limit of detection (LOD), the limit of quantification (LOQ), precision, accuracy, and robustness as per ICH guidelines on both the columns.

\subsubsection{Specificity:}

Specificity study was conducted for the interference of any other peak at the retention time of the enantiomers peak in blank solution. Diluent was injected as a blank solution. Both peaks were well separated and there was no interference from blank. All system suitability parameters were as per norms. The system suitability parameters are shown in Table 6. 


\subsubsection{Linearity}

The working standard solutions for linearity determination were prepared by diluting the stock solution of racemic milnacipran to the required concentrations in the mobile phase. These solutions were injected three times and peak areas were recorded. A linearity curve was plotted between peak areas versus analyte concentration for both the enantiomers. After linearity was plotted, the slope, y-intercepts, correlation coefficient and regression coefficient $\left(\mathrm{r}^{2}\right)$ was determined. The linearity equation for Levo-Mil and Dex-Mil is $y=25453 x+82775$ and $y=25355 x+85118$ respectively. Good correlation coefficient $\left(\mathrm{r}^{2}\right)=0.9991$ was obtained for both the enantiomers.

\subsubsection{Precision}

The precision of the method was performed by repeatability and intermediate precision studies. The repeatability of the method was determined by making 3 injections at three different concentration level $(15,30$ and $45 \mu \mathrm{g} / \mathrm{ml})$ of working standard solutions of racemic milnacipran hydrochloride and \%RSD of peak area was calculated for each of the enantiomers. The intermediate precision of the method was performed by intra-day precision, inter-day precision, and different instrument. For intermediate precision, 6 determinations of working standard solution of racemic milnacipran were carried out and \%RSD of peak area was calculated for each of the enantiomers. \% RSD of replicate injections in repeatability and intermediate precision was found to be less than $2 \%$ for both enantiomers.

\subsubsection{Accuracy}

The accuracy of the method was assessed by conducting recovery studies. Recovery studies for Levo-milnacipran were conducted at three concentration levels i.e. at 50\% (7.5 $\mu \mathrm{g} / \mathrm{ml}), 100 \%(15$ $\mu \mathrm{g} / \mathrm{ml})$ and $150 \%(22.5 \mu \mathrm{g} / \mathrm{ml})$ of target concentration. Each concentration level was analyzed in triplicate and \% recovery was calculated. The \% recovery for both enantiomers was found to be from $\mathbf{9 9 - 1 0 1 \%}$. The results are shown in Table 5.

Table 5: Recovery results

\begin{tabular}{|c|c|c|}
\hline $\begin{array}{c}\text { Actual concentration of Levo- } \\
\text { milnacipran }(\boldsymbol{\mu g} / \mathbf{m l})\end{array}$ & $\begin{array}{c}\text { Recovered concentration } \\
(\boldsymbol{\mu g} / \mathbf{m l}), \text { Mean } \mathbf{\pm} \text { SD }\end{array}$ & $\begin{array}{c}\text { \% Recovery } \\
\text { Mean } \mathbf{\mathbf { S D }}\end{array}$ \\
\hline 7.5 & $7.52 \pm 0.19$ & $99.05 \pm 0.53$ \\
\hline 15 & $15.2 \pm 0.14$ & $101.53 \pm 0.95$ \\
\hline 22.5 & $22.4 \pm 0.17$ & $99.68 \pm 0.76$ \\
\hline
\end{tabular}




\subsubsection{Limit of detection and quantification}

The LOD and LOQ were determined by injecting a series of dilute solutions. LOD and LOQ were calculated on the basis of the response and slope of the regression equation. They were calculated from the formula $3.3 \sigma / \mathrm{s}$ and $10 \sigma / \mathrm{s}$ respectively where ' $\sigma$ ' is the standard deviation of the $y$ intercept of the regression line and ' $S$ ' slope of the calibration curve. The limit of detection and limit of quantification was found to be $0.125 \mu \mathrm{g} / \mathrm{ml} \& 0.5 \mu \mathrm{g} / \mathrm{ml}$ respectively for both enantiomers.

\subsubsection{Robustness}

To determine the robustness of the method, deliberate changes were made in the experimental conditions of the developed method. The resolution between two enantiomers and system suitability parameters were evaluated. The variables evaluated in the study were flow rate $( \pm 0.1$ $\mathrm{ml} / \mathrm{min}$.), $\mathrm{pH}$ of mobile phase $( \pm 0.2)$, Mobile phase composition ( \pm 2 Units) and variation in detection wavelength $( \pm 2 \mathrm{~nm})$. The robustness was determined from triplicate injections of racemic milnacipran hydrochloride. In all cases, the resolution was greater than 2.0, \%RSD of peak area was less than 2 and the tailing factor was less than 1.5 for both the enantiomers.

\subsection{Application of the method}

The validated HPLC method was used for the quantitative analysis of Levo-milnacipran hydrochloride in pharmaceutical formulation (Fetzima). The assay chromatogram of capsule formulation is shown in Figure 5. The assay of Levo-milnacipran hydrochloride in the marketed formulation is presented in Table 6 .

Table 6: Assay of Levo-milnacipran hydrochloride in pharmaceutical formulation

\begin{tabular}{|l|l|c|c|c|c|}
\hline Formulation & $\begin{array}{l}\text { Label Claim } \\
(\mathbf{m g})\end{array}$ & \multicolumn{2}{|c|}{$\begin{array}{c}\text { Amount estimated (mg) } \\
\text { mean }\end{array}$} & \multicolumn{2}{c|}{$\%$ Assay } \\
\cline { 3 - 6 } & & Levo- Mil & Dex- Mil & Levo- Mil & Dex-Mil \\
\hline Fetzima & 120 & 120.4 & - & 100.3 & - \\
\hline
\end{tabular}

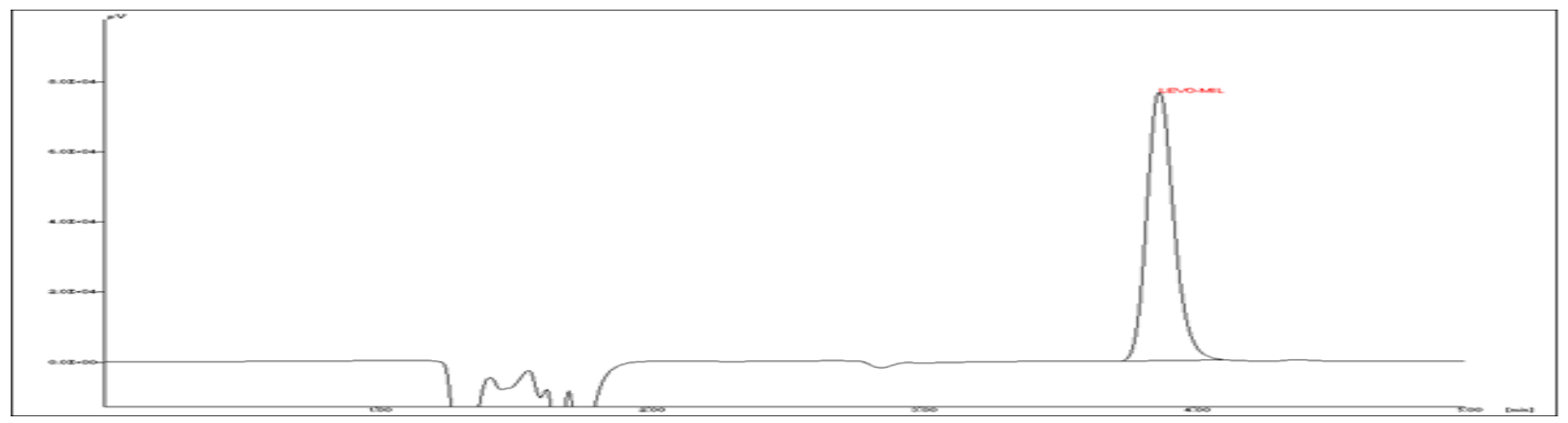

Figure 5: Assay of Levo-milnacipran formulation 


\subsection{Molecular docking to estimate complexation stability}

An attempt was made to understand the binding of enantiomers with chiral selectors by employing molecular docking. The details, at atomistic levels, about the interactions between $S \beta C D$ and each enantiomer of milnacipran hydrochloride were further sought using molecular docking. The GLIDE Emodel energy was used as the yardstick to rank order the complexes. It must be noted that the enantiomer with the best Emodel score is expected to elute before the one with a lower Emodel score. Here, we observed that Levo-milnacipran with Emodel energy $-42.3 \mathrm{kcal} / \mathrm{mol}$ forms a better complex with S $\beta C D$ than Dextro-milnacipran with Emodel energy $-38.9 \mathrm{kcal} / \mathrm{mol}$. Hence docking studies suggest that Levo-milnacipran will be eluted before Dextro-milnacipran. Our docking studies corroborate with the experimental observations. The inclusion complexes of S $\beta C D$ with Dextro-milnacipran and Levo-milnacipran are depicted in Figure 6.
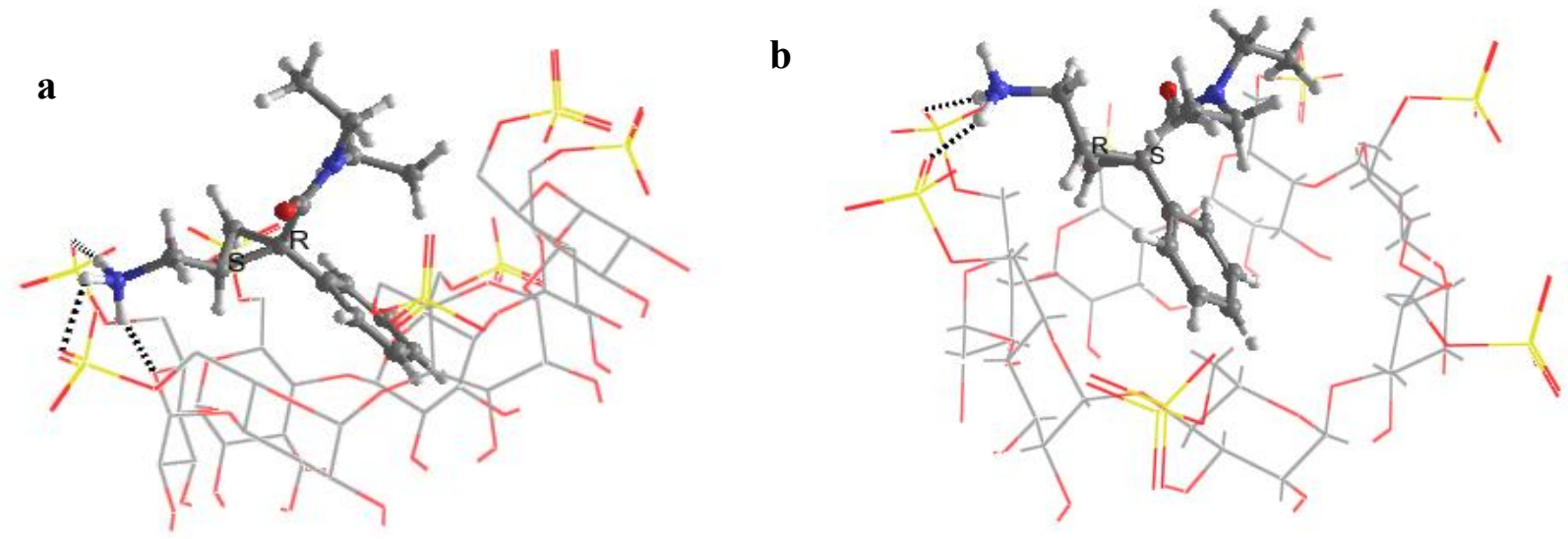

Figure 6: Docked structure of S- $\beta-C D$ with (a) Dextro-milnacipran and (b) Levo-milnacipran

\subsection{Comparison with reported methods:}

As mentioned earlier, there are methods reported for the separation of milnacipran enantiomers by chiral columns. JiaYan LE et al [15] have reported enantio-separation of milnacipran by HPLC method with hydroxypropyl $\beta$-cyclodextrin-based chiral stationary column Astec CYCLOBOND ${ }^{\mathrm{TM}}$ I $2000 \mathrm{RSP}$ column $(250 \mathrm{~mm} \times 4.6 \mathrm{~mm}, 5 \mu \mathrm{m})$ the mobile phase was acetonitrile-0. $1 \%$ triethylamine acetate $(\mathrm{pH}$ 6. 0) $(5: 95, \mathrm{~V} / \mathrm{V})$ and the resolution was 1.63. Yifeng, $C$ et al [16], have reported enantio-separation of milnacipran by HPLC method with $\beta$ cyclodextrin-based chiral column Astec CYCLOBOND ${ }^{\mathrm{TM}}$ I 2000 AC $(25 \mathrm{~cm} \times 4.6 \mathrm{~mm}, 5 \mu \mathrm{m})$, the mobile phase was acetonitrile- $0.1 \%(\mathrm{v} / \mathrm{v}) \mathrm{pH} 5.0$ triethylamine acetate (TEAA) $(5: 95, \mathrm{v} / \mathrm{v})$ with a flow rate of $0.4 \mathrm{~mL} / \mathrm{min}$ and the detection wavelength was $220 \mathrm{~nm}$. The injection volume was 
$10 \mu \mathrm{L}$ and the column temperature was $25^{\circ} \mathrm{C}$. The value of the resolution $\left(R_{\mathrm{s}}\right)$ was 1.74 with a runtime of 10minutes. The method reported in this paper is the RP-HPLC method with sulfated $\beta$ cyclodextrin as CMPA on achiral core-shell column gave a resolution of 2.96 in the run-time of 5 minutes, indicating that this developed method is superior to the reported chiral separation methods for milnacipran. Hence CMPA technique should be evaluated alongwith CSP technique.

\section{Conclusion:}

A cost-effective, validated, simple, fast RP-HPLC method with a good resolution was developed, using sulfated $\beta$-cyclodextrin for chiral separation of milnacipran enantiomers. The simplicity of the method is attributed to the fact that there was no need for any derivatization. Furthermore, it excludes the use of various expensive chiral stationary phases and instead makes use of easily available RP-HPLC $\mathrm{C}_{8}$ columns. Commercially available cyclodextrin bonded chiral columns have preloaded unadjustable amount of cyclodextrin. As shown in this paper, the concentration of cyclodextrin in the mobile phase is the most important parameter governing enantiomeric resolution. If sufficient cyclodextrin is not present on the column, there will not be complete formation of transient diastereomeric inclusion complex and subsequently, satisfactory resolution may not be obtained. The key step in achieving good chiral separation is the formation of stable inclusion complex between milnacipran hydrochloride and S $\beta C D$. It was observed that inclusion complex formation stabilized by hydrogen bonding is a major force responsible for the chiral separation, in this case. Out of two achiral columns investigated, the column packed with coreshell particles outperformed the fully porous column. With the help of the core-shell column, there was not only a reduction in analysis time but also a reduction in consumption of costly chiral selector, hence making separation quick and economic.

\section{Acknowledgments}

The authors are thankful to the University Grants Commission (F.NO.- 41-721/2012, Multicomponent Analysis of Drugs), New Delhi and Department of Science and Technology (DST-DPRP: Project sanction number: VI-D\&P/552/2016-17/TDT(G) dated 07.03.2017), New Delhi for funding this project. We are also thankful to Arch Pharma Labs (Mumbai, India) for

providing racemic milnacipran hydrochloride and levomilnacipran hydrochloride and Dr. Ravi Shanker Kovi, Apicore US LLC., for providing Levomilnacipran formulation (Fetzima). Further, 
the authors wish to acknowledge Signet Chemicals (Mumbai, India) for providing $\beta$-cyclodextrin and Hydroxypropyl- $\beta$ - cyclodextrin.

\section{References:}

[1] Kanchan Nautiyal, K. Ramakrishna. Method development and validation for determination and quantitative estimation of impurities in milnacipran hydrochloride by liquid chromatography technique. J. Chem. Pharm. Res 2014; 6(10): 358-366

[2] Matthew Macoluso, Hala Kazanchi, Vikram Malhotra. Levomilnacipran for the treatment of major depressive disorder. Current Psychiatry 2013; 12 (12): 50-52.

[3] FDA's Policy Statement for the Development of New Stereoisomeric Drugs. Chirality, 1992; 4(5), pp. 338-40.

[4] J. Caldwell, Importance of stereospecific bioanalytical monitoring in drug development, $J$ Chromatogr A, 719 (1996) 3-13.

[5] L. Coventry. Chiral Liquid Chromatography. Chapman \& Hall, New York, NY, 1989, 148-49.

[6] W. C. Purdy and S. Li. Cyclodextrins and their applications in analytical chemistry.

Chem. Rev. 92 (1992) 1457-70.

[7] Shengli Ma, Sherry Shen, Nizar Haddad, Wenjun Tang, JingWang, Heewon Lee, Nathan Yee, Chris Senanayake, Nelu Grinberg, Chromatographic and spectroscopic studies on the chiral recognition of sulfated $\beta$-cyclodextrin as chiral mobile phase additive Enantiomeric separation of a chiral amine, J Chromatogr A. 1216 (2009) 1232-1240

[8] Chen Zhang, Wei X. Huang, Zhi Chen, Abu M. Rustum, Separation of chiral primary amino compounds by forming a sandwiched complex in reversed-phase high performance liquid chromatography. J Chromatogr A, 1217 (2010) 4965-4970.

[9] R. J. Hurtubise and R. M. Mohseni. Retention characteristics of several compound classes in reversed-phase liquid chromatography with $\beta$-cyclodextrin as a mobile phase modifier. $J$. Chromatogr. 499 (1990) 395-410.

[10] Magdalena Taschwer, Yvonne Seidl, Stefan Mohr, and Martin G. Schmid. Chiral separation of cathinone and amphetamine derivatives by HPLC/UV using sulfated $\beta$-cyclodextrin as chiral mobile phase additive. Chirality 2014; 26: 411-418.

[11] Jin Xu, Wenxiu Zhao, Yawei Ning, Mohanad Bashari, Yisheng Chen, Zhengyu Jin, Na Yang, Xueming Xu. Enantiomer separation of phenyllactic acid by HPLC with Hp- $\beta$-cyclodextrin as chiral mobile phase additive. J Incl Phenom Macro 2013; 76: 461-465. 
[12] Jincui Ye, Guosheng Chen, Su Zeng. Enantiomeric separation of norgestrel by reversed phase high performance liquid chromatography using eluents containing hydroxypropyl $\beta$-cyclodextrin in stereoselective skin permeation study. J Chromatogr B; 843(2006) 289-294.

[13] Surendra Dutt Sharma, Gaurav Singh. Enantioseparation of nadifloxacin by high performance liquid chromatography. Adv Anal Chem 2012; 2(4): 25-31.

[14] Zhefeng Zhang, Gengliang Yang, Dexian Wang, Guijian Liang \& Yi Chen. Chiral separation and enantiomeric purity determination of pazufloxacin mesilate by HPLC using chiral mobile phase additives. J Liq Chromatogr R T, 2005; 27(5): 813-827.

[15] CHEN, XiuJuan \& ZHENG, Zhen \& ZHANG, MingYong \& LE, JiaYan \& LI, WuHong \& HONG, ZhanYing. Enantioseparation of milnacipran by HPLC method with hydroxypropyl $\beta$ cyclodextrin-based chiral stationary column. Pharmaceutical Care and Research; 2017, 17(3):210212.

[16] Zhen, Z., Xiujuan, C., Liang, Z., Wuhong, L., Zhanying, H. and Yifeng, C., Enantioseparation of milnacipran enantiomers and the separation mechanism on beta-cyclodextrin-based chiral stationary phases by high performance liquid chromatography. CHINESE JOURNAL OF CHROMATOGRAPHY, 2017, 35(3), pp.286-290.

[17] Angela Patti, Sonia Pedotti, Claudia Sanfilippo. Chiral HPLC analysis of milnacipran and its FMOC-derivative on cellulose-based stationary phases. Chirality; 2008; 20: 63-68.

[18] Marie Lecoeur-Lorin, Rapha Delepee, Jean-Paul Ribet, Philippe Morin, Chiral analysis of milnacipran by a nonchiral HPLC- circular dichroism: Improvement of the linearity of dichroic response by temperature control. J. Sep. Sci. 2008, 31, 3009 - 3014.

[19] Preti, R., 2016. Core-shell columns in high-performance liquid chromatography: Food analysis applications. International journal of analytical chemistry, 2016. 